FTUAM 97-1

UM-TH-97-15

June, 1997

\title{
Dynamically Generated Masses in Supersymmetric QCD and Quark Mass Problems*
}

\author{
F. J. Ynduráin \\ Randall Lab of Physics \\ University of Michigan, \\ Ann Arbor, MI -48106, USA \\ and \\ Departamento de Física Teórica, C-XI, \\ Universidad Autónoma de Madrid, \\ Canto Blanco, \\ E-28049, Madrid, Spain. \\ e-mail: fjy@delta.ft.uam.es
}

\begin{abstract}
We consider possible mechanical masses that could appear in supersymmetry, other than by direct Higgs coupling to fermions and we speculate that the existence of such a type of mass would allow one to have the Higgs mass of the $u$ quark zero, and the Higgs mass of the $d$ quark (at $1 \mathrm{GeV}$ ) of $\approx 1$ to $2 \mathrm{MeV}$, thus solving at the same time the strong CP problem and arranging the grand unification prediction

$$
m_{\mu} / m_{e}=m_{s, \text { Higgs }} / m_{d, \text { Higgs }} .
$$

One possible mechanism for this is related to, but not identical with the quark condensate. Here a mass is generated which is the same for all quarks, and which adds to the Higgs type mass. Unfortunately, the numerical value of the generated mas falls short of the desired value (some $5 \mathrm{MeV}$ ) by orders of magnitude.

An alternate mechanism, through Higgs-induced left-right couplings in the squark sector may produce masses of the correct order of magnitude if the mixing angles are diferent in the squark and quark sectors. To get the desired result for the $u$ quark mass, we need a stop component mixing of $1 / 20$ for the $L R$ $\tilde{u}$ squark coupling, so the strong CP problem may still be solved. For the $d$ mass, this mechanism is not really sufficient to solve the grand unification mass ratio problem.
\end{abstract}

\footnotetext{
* Typeset with $\mathrm{P}_{\mathrm{H}} \mathrm{ys}^{\mathrm{M}} \mathrm{TE}_{\mathrm{E}} \mathrm{X}$
} 


\section{-F. J. YNDURÁIN-}

As is well known, chiral symmetry is dynamically broken in QCD through the nonzero value of the quark condensate:

$$
\langle\bar{\psi} \psi\rangle=\langle\operatorname{vac}|: \bar{\psi}(0) \psi(0):| \operatorname{vac}\rangle \neq 0 .
$$

where $\mid$ vac $\rangle$ is the physical vacuum. This phenomenon occurs even for massless quarks. The quantity $\langle\bar{\psi} \psi\rangle$, while presenting some of the properties of a mass term, does not imply the generation of a mechanical mass. This is easily seen by noticing that insertion of $\langle\bar{\psi} \psi\rangle$ in a Green's function (for example, the quark propagator) with momentum $q$ yields contributions behaving like

$$
q^{-4}\langle\bar{\psi} \psi\rangle
$$

for large $q$, while a mechanical mass would give

$$
m q^{-2}
$$

In this note we remark that, in supersymmetric QCD, broken only by a mass term for squarks and gluinos, the quark condensate does generate a mass. This mass behaves as a mechanical mass up to energies comparable to the squark, gluino masses, that we take for simplicity to be of the same order of magnitude,

$$
m_{\text {gluino }} \approx m_{\text {squark }} \approx \tilde{m} .
$$

An alternate mechanism for the generation of masses for quarks occurs if there is a mismatch between the mixing (Cabibbo-Kobayashi-Maskawa) angles for quarks and squarks. This would mean that the Higgs-generated mass type terms,

$$
-\delta_{f}^{2} \phi_{L f}^{+} \phi_{R f}+\text { h.c. }
$$

(the $\phi$ represent squarks, and $f$ is a flavour index) get contributions from several flavours. Thus, even if the Higgs-up quark coupling would be zero, $\delta_{u}$ could have a nonzero value due to mixing, in particular of squarks $\tilde{u}$ and $\tilde{t}$. One then expects,

$$
\delta_{u} \cong\left(\sin \tilde{\theta}_{u t}\right) m_{t}, \delta_{d} \cong\left(\sin \tilde{\theta}_{d b}\right) m_{b} .
$$

We explore possible connections with phenomenological issues.

\section{Dynamically Generated Quark Mass}

We take our model to be given by the Lagrangian (for simplicity we consider here only one flavor),

$$
\begin{gathered}
\mathcal{L}=\overline{\psi_{L}} \mathrm{i} \gamma \cdot D \psi_{L}+\overline{\psi_{R}} \mathrm{i} \gamma \cdot D \psi_{R}-(1 / 4) G^{2}+\bar{\lambda}\left(\mathrm{i} \gamma \cdot D-m_{\lambda}\right) \lambda \\
+g \sqrt{2}\left(\phi_{L} \overline{\psi_{L}}+\phi_{R} \overline{\psi_{R}}\right) \lambda+\text { h.c. }+\phi_{L}^{+}\left(\square+\mu^{2}\right) \phi_{L}+\phi_{R}^{+}\left(\square+\mu^{2}\right) \phi_{R} .
\end{gathered}
$$

Note that as yet we do not introduce by hand off-diagonal mass terms

$$
\delta^{2}\left(\phi_{L}^{+} \phi_{R}+\phi_{R}^{+} \phi_{L}\right)
$$

so $\mathcal{L}$ is invariant under independent chiral transformations of all the $L$ and $R$ fields.

The mechanism for generation of the non-Higgs mechanical mass is simple. Consider the left-right piece of the quark propagator,

$$
S=\left\langle\operatorname{vac}\left|\mathrm{T} \psi_{L} \bar{\psi}_{R}\right| \mathrm{vac}\right\rangle .
$$

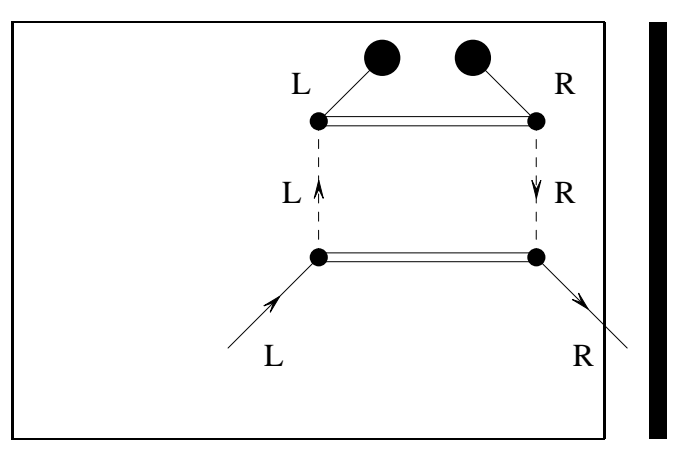

FIGURE 1. Diagram yielding mass-like term from the quark condensate. 
The relevant effect is generated by the diagram of Fig. 1. The large blobs there represent the vacuum expectation value of the quark condensate. The corresponding contribution to $S, S_{m}$ is evaluated with standard techniques. One gets the result

$$
\begin{array}{r}
S_{m}=\left(-1 / 3 p^{2}\right) \mathrm{i} g^{4}\left(1+\gamma_{5}\right)\left(C_{F}^{2} / N_{c}\right) m_{\lambda}^{2} \\
\times \int \frac{\mathrm{d}^{4} k}{(2 \pi)^{4}} \frac{1}{(p-k)^{2}-m^{2}} \frac{1}{\left(k^{2}-\mu^{2}\right)^{2}} \frac{1}{k^{2}-m_{\lambda}^{2}}\langle\bar{\psi} \psi\rangle \\
\approx i \frac{-\alpha_{s}^{2} C_{F}^{2}}{3 p^{2} N_{c}}\left(1+\gamma_{5}\right) \frac{\langle\bar{\psi} \psi\rangle}{6 \tilde{m}^{2}},
\end{array}
$$

the last expression for $\left|p^{2}\right| \ll m_{\lambda} \approx \mu \approx \tilde{m}$. This corresponds, for momenta $q^{2}$ such that $\Lambda^{2} \ll\left|q^{2}\right| \ll \tilde{m}^{2}$ (with $\Lambda$ the QCD parameter) to a generated mechanical mass

$$
m_{\bar{\psi} \psi}=\frac{2 C_{F}^{2} \alpha_{s}^{2}}{9 N_{c}} \frac{-\langle\bar{\psi} \psi\rangle}{\tilde{m}^{2}} .
$$

\section{Applications}

Eqs. (2), (3) have been obtained for quarks with, initially, zero mechanical mass. If we take a quark with non-zero mass $m_{\text {Higgs }}$ generated by the Higgs mechanism, with $m_{\text {Higgs }} \ll \Lambda$, then we get a total mass

$$
m=m_{\text {Higgs }}+m_{\bar{\psi} \psi} \text {. }
$$

The existence of a mass generated by a mechanism other than the Higgs one would be welcome for at least two reasons. To discuss them, we first remark that all experimentally based determinations of the masses of the light quarks ${ }^{[1,2]}$ yield the total mass, $m$. From the standard values of the $\overline{\mathrm{MS}}$-renormalized masses, at the reference momentum of $1 \mathrm{GeV}$, we then have

$$
m_{u}=5 \mathrm{MeV}, m_{d}=8 \mathrm{MeV}, m_{s}=200 \mathrm{MeV} .
$$

Now, we can assume that all the $u$ quark mass is generated dynamically ${ }^{1}$. Thus, the Higgs mass of this quark would vanish (apparently, this occurs naturally in a class of supersymmetric models ${ }^{[3]}$ ) and we would preserve the two independent chiral invariances $\mathrm{U}(1)_{L} \times \mathrm{U}(1)_{R}$, which is sufficient to solve the strong $\mathrm{CP}$ problem. This assumption on the mass of the $u$ quark then implies

$$
m_{\bar{\psi} \psi}=5 \mathrm{MeV}
$$

and therefore we get the second bonus: from this and the values of the masses in Eq.(4) it follows that

$$
m_{d, \text { Higgs }}=3 \mathrm{MeV} \text {, }
$$

which goes in the righ direction towards solving the outstanding mass puzzle ${ }^{[4]}$ that occurs in grand unified $\mathrm{SU}(5)$, with minimal Higgs system. It will be remembered that there one has

$$
m_{b, \text { Higgs }}=m_{\tau}
$$

a relation well satisfied if taking into account renormalization effects. However, the same assumptions give

$$
m_{s} / m_{d}=m_{\mu} / m_{e}
$$

This relation is independent of renormalization effects, and is violated by a full order of magnitude if we take the masses in (5) to mean the full masses: note that Eq. (4) gives

$$
m_{s} / m_{d}=25 .
$$

However, relation (5) actually follows for the Higgs masses, and then

$$
m_{s, \text { Higgs }} / m_{d, \text { Higgs }}=67,
$$

1 From the existence of lower bounds on quark masses, independent on assumptions about experimentally inaccessible pieces of the pseudoscalar correlator (ref. 2) it follows that the possibility $m_{u}=0$ cannot be contemplated seriously as a solution to the strong $\mathrm{CP}$ problem. 


\section{-F. J. YNDURÁIN-}

and by fiddling with the errors of (4) one can even get to the experimental $\mu$ /e ratio.

\section{Discussion}

The previous analysis has made it clear that it would be very desirable to have, beyond the Higgs mass, a dynamically generated mass of the order of $m_{u}$. The mechanism discussed at the begining of this note provides such a mass. Unfortunately, it is far too small. If we assume $\tilde{m} \approx 100 \mathrm{GeV}$, and renormalize at $100 \mathrm{GeV}$, then we get

$$
m_{\bar{\psi} \psi} \approx 2.1 \times 10^{-6} \mathrm{MeV}
$$

way off the necessary

$$
m_{u}(100 \mathrm{GeV}) \approx 2.7 \mathrm{MeV} .
$$

It is of course possible to get the desired effects by introducing, in an ad hoc manner, a condensate

$$
\left\langle\phi_{L}^{+} \phi_{R}\right\rangle \neq 0
$$

or, equivalently, by assuming a coupling

$$
-\delta^{2} \phi_{L}^{+} \phi_{R}+\text { h.c.. }
$$

Let us consider the second for definiteness, and because it arises somewhat naturally in the standard supersymmetric context. Assume again degenerate SUSY masses $\tilde{m}$ for simplicity. The term (6) still respects the two independent $\mathrm{U}(1)_{L} \times \mathrm{U}(1)_{R}$, for fermions: this is sufficient for our aims. The mass generated, given by the diagram of Fig. 2, is easily computed, and we get, for momenta much smaller than $\tilde{m}$,

$$
m_{\phi_{L}^{+} \phi_{R}}=\frac{\delta^{2} \alpha_{s}\left(\tilde{m}^{2}\right)}{4 \pi \tilde{m}} .
$$

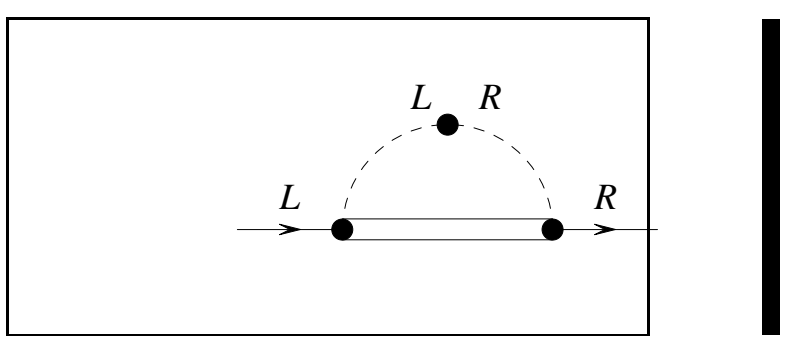

FIGURE 2. Diagram yielding mass term from $L R$ squark coupling.

For $\tilde{m}=200 \mathrm{GeV}$, agreement with the phenomenological value of the $u$ quark mass, still with with zero Higgs mass, is obtained if $\sqrt{\delta_{u}^{2}} \simeq 8 \mathrm{GeV}$, i.e., $\sin \tilde{\theta}_{u t} \sim 1 / 20$.

This mechanism therefore appears to give a possible solution to the strong CP problem, although it looks a bit contrived: there seems to be no apparent reason why the angle $\tilde{\theta}_{u t}$ should be so much larger than the corresponding quark mixing angle, $O\left(10^{-3}\right)$. The problem of the mass ratio, Eq. 5 , is however left unsolved. To get a contribution to the phenomenological value of the $d$ quark mass so that its Higgs mass is left with only $\sim 2 \mathrm{MeV}$ one also needs $\sqrt{\delta_{d}^{2}} \simeq 8 \mathrm{GeV}$, which is impossible as this is larger than the bottom quark mass. One could try to repair this with a mixed mechanism: it is possible to imagine that the electron observed mass is now the difference between a larger Higgs mass, and a SUSY generated mass, due to mixing of the Higgs couplings of $\tilde{e}$ and $\tilde{\tau}$, so one would have $m_{e}=m_{e, \text { Higgs }}-m_{e, \phi^{+} \phi}$. Renormalizing the masses at the SUSY scale, $\tilde{m} \simeq 200 \mathrm{GeV}$, we have

$$
\begin{array}{r}
m_{b} \simeq 3 \mathrm{GeV}, m_{\tau} \simeq 2 \mathrm{GeV}, \alpha_{s} \simeq 0.1 \\
\text { and } \\
m_{e}=0.5 \mathrm{MeV}, m_{d}=4.5 \mathrm{MeV}, m_{s}=150 \mathrm{MeV}
\end{array}
$$

so that use of Eq. (7) for the Higgs components gives

$$
\frac{m_{d, \text { Higgs }}}{m_{e, \text { Higss }}}, m_{\phi^{+} \phi} \simeq 2.5 \mathrm{MeV},
$$

which is hardly possible, even with mixing angles of $\tilde{\delta}_{b} \simeq \tilde{\delta}_{\tau} \simeq \pi / 2$-quite an extreme situation indeed.

In summary, we may say that the mechanism that is natural does not work phenomenologically, and the one that works phenomenologically lacks sufficient theoretical justification. The results may be 
-DYNAMICALLY GENERATED MASSES-

relevant to the strong $\mathrm{CP}$ problem, but less likely so for the unification of fermion masses: certainly not unless unexpected physics enter the game. 
-F. J. YNDURÁIN-

Acknowledgements

Discussions with G. Kane on possible supersymmetric scenarios are also gratefully ackowledged.

\section{References}

1. J. Gasser and H. Leutwyler, Phys. Rep., C87 (1982) 77; C. A. Domínguez And E. De Rafael, Ann. Phys. (N.Y.), 174 (1987) 372.

2. C. Becchi et Al.,Z. Phys., C8 (1981) 375

3. See, e.g., P. Binetruy, S. Laynac And P. Ramond, Nucl. Phys., BB477 (1996) 353, and work quoted there.

4. F. J. Ynduráin, Kinam, 1 (1979) 167. 